\title{
Increased emergency department chief complaints of fever identified the influenza (H1N1) pandemic before outpatient symptom surveillance
}

\author{
Zvi Shimoni · Joseph Rodrig • Natan Dusseldorp • \\ Mark Niven · Paul Froom
}

Received: 2 December 2010/Accepted: 11 March 2011/Published online: 30 March 2011

(C) The Japanese Society for Hygiene 2011

\begin{abstract}
Objective To determine whether a sentinel clinic network or an emergency department (ED) was more timely in identifying the 2009 influenza A (H1N1) pandemic.

Methods All reasons for presenting to the adult regional medical ED were coded online by admission secretaries, without the aid of medical personnel. Increased influenza activity defined by weekly chief complaints of fever was compared with activity defined by the Israel Center for Disease Control (viral surveillance as well as a large sentinel clinic network).

Results Influenza activity during the pandemic increased in the ED 2 weeks before outpatient sentinel clinics. During the pandemic, maximal ED activity was much higher than in previous seasons. Maximal activity during the past 5 years correlated with the timeliness of the chief complaint of fever in identifying the onset of epidemics.
\end{abstract}

\footnotetext{
Z. Shimoni - M. Niven

Department of Internal Medicine B,

Laniado Hospital, Netanya, Israel

J. Rodrig

Emergency Department, Laniado Hospital, Netanya, Israel

N. Dusseldorp

Computer Support Department, Laniado Hospital,

Netanya, Israel

P. Froom

Department of Epidemiology and Preventive Medicine,

School of Public Health, Tel Aviv University, Ramat Aviv, Israel

P. Froom ( $\square)$

Maagan Michael, DN Menashe 37805, Israel

e-mail: froomp@gmail.com
}

Conclusion Chief complaint of fever in the ED can be a sensitive marker of increased influenza activity and might replace the use of sentinel clinics.

Keywords Emergency department · Fever · Clusters . Influenza $\cdot$ Epidemics

\section{Introduction}

Timely regional monitoring of influenza-related morbidity is a priority for seasonal surveillance and pandemic preparedness [1,2], since public health intervention strategies can be beneficial [2]. Methods to identify epidemics include obtaining data from over-the-counter drug sales, sentinel practices, absenteeism records, telephone triage centers, home visits by general practitioners, health advice calls, and emergency departments [2-13]. More recently, identification of epidemics based on data from the web has been reported with promising results in studies using search engines [14-16].

Due to time and budget constraints, surveillance tends to be restricted in scale. Even in developed countries, information from sentinel clinics may be difficult to obtain, and commonly there is a low and variable participation rate, with reporting delays limiting usefulness [2]. Emergency department (ED) data on the other hand is a more convenient data source with higher participation rates [2].

In the ED, previous attempts to identify increased influenza activity included triage nurses recording complaints by categories [5], syndromic analysis of computerized emergency department patients' chief complaints [6], and patient-based free text grouped into diagnostic groups $[2,7]$. We have previously shown that the frequency of a chief complaint of fever identified periods of 
increased influenza activity in Israel without false-positive periods [8], yet there was a delay of a few weeks between an increase of influenza-like symptoms reported in outpatient clinics and the onset of the epidemic as defined by a chief complaint in the ED. This delay might have been because of the relatively small size of the ED, and sensitivity might be higher in settings with more patients, such as in ED networks.

In Israel the onset of the pandemic H1N1 influenza outbreak was clearly defined because of early warnings of the approaching pandemic. No-one predicted that the pandemic would begin in Mexico in mid-April and then during the following months spread to North America, Europe, and Israel. In Israel the first imported cases were detected in week 21 of 2009 and the first local cases at the end of week 22. However, an increase in influenza-like symptoms (ILS) reported outpatient clinics occurred first only by week 26 [17]. We hypothesized that the frequency of fever in the ED would identify the onset of the Israeli epidemic at least as early as the increase in ILS reported in outpatient clinics.

\section{Methods}

Laniado Hospital serves a population of around 200,000, but patients can choose to go to hospitals outside the region. All reasons for presenting to the adult medical ED were coded online by admission secretaries, without the aid of medical personnel, over a 5-year period. The patient or family member is asked why the patient came to the ED. There were no missing values, since the code must be entered in order to admit the patient to the emergency department. The secretary chose from a list of 102 reasons according to the ICD-9, with the addition of a few complaints such as paralysis, hypertension, and the diagnosis of asthma.

During the H1N1 pandemic and 4 previous years, increased influenza activity and the maximal week of influenza activity were defined by ILS from sentinel clinics and maximal proportion of positive culture results [17]. We defined any 7 -day period with at least 45 chief complaints of fever as representing increased influenza activity, since there was only one isolated week with such findings outside the Israel Center for Disease Control-defined increased influenza activity weeks during that time period.

\section{Results}

During the 4 years preceding the pandemic there was a delay in increased activity in the ED when compared with outpatient clinics (Table 1), and maximal activity correlated with the timeliness of the chief complaint of fever in identifying the onset of epidemics. The two epidemics with the lowest maximum ED visits had prolonged delays. The delay was 9 weeks in 2005-2006, and in the 2008-2009 epidemic no increased activity was found in the ED. The delay in 2007-2008 was 4 weeks, and in 2006-2007 (the year with the highest activity before 2009-2010) it was only 2 weeks. However, during the pandemic, maximal ED activity was much higher than the other seasons (Table 1), and increased activity in the ED preceded the ILS outpatient activity by 2 weeks (Figure 1).

\section{Discussion}

The major finding of our study is that, during the H1N1 pandemic, an increase in ED activity occurred before an increase in ILS activity in the outpatient clinics. Excess numbers of patients presenting to the ED with fever predicted 3 of the 4 epidemics during the previous 4 years, with one isolated false-positive week. There was, however, a delay of a few weeks from increased outpatient activity until identification in ED patients. This was not the case however for 2009-2010, when the increase in ED activity preceded that of the outpatient clinics and occurred about 1 week after the first local cases were discovered. This suggests that the sensitivity of this methodology is dependent on the number of ill patients seen in the ED and that it is possible that this methodology could replace outpatient clinics if a single department or network of departments was large enough.

The establishment of a large network of emergency departments is practical. In New York City a study showed compliance rates of up to $90 \%$ [2]. They used a complicated system of extracting key words from patients' recorded chief complaint. Our study suggests that fever as a single symptom should be examined in such settings and in other settings using various methodologies.

Studies outside the emergency department setting support our findings. A study in England of spatiotemporal

Table 1 Onset of epidemic (week of year): influenza-like symptoms (ILS) activity in the community and chief complaints of fever in the emergency department (ED)

\begin{tabular}{|c|c|c|c|}
\hline \multirow[t]{2}{*}{ Year } & \multicolumn{2}{|c|}{ Onset week } & \multirow{2}{*}{$\begin{array}{l}\text { Maximum ED fever } \\
\text { visits-1 week } \\
\mathrm{N}\end{array}$} \\
\hline & ILS & ED & \\
\hline 2005-2006 & 1 & 10 & 52 \\
\hline 2006-2007 & 49 & 51 & 76 \\
\hline 2007-2008 & 51 & 3 & 65 \\
\hline 2008-2009 & 48 & None & 44 \\
\hline 2009-2010 & 26 & 24 & 101 \\
\hline
\end{tabular}




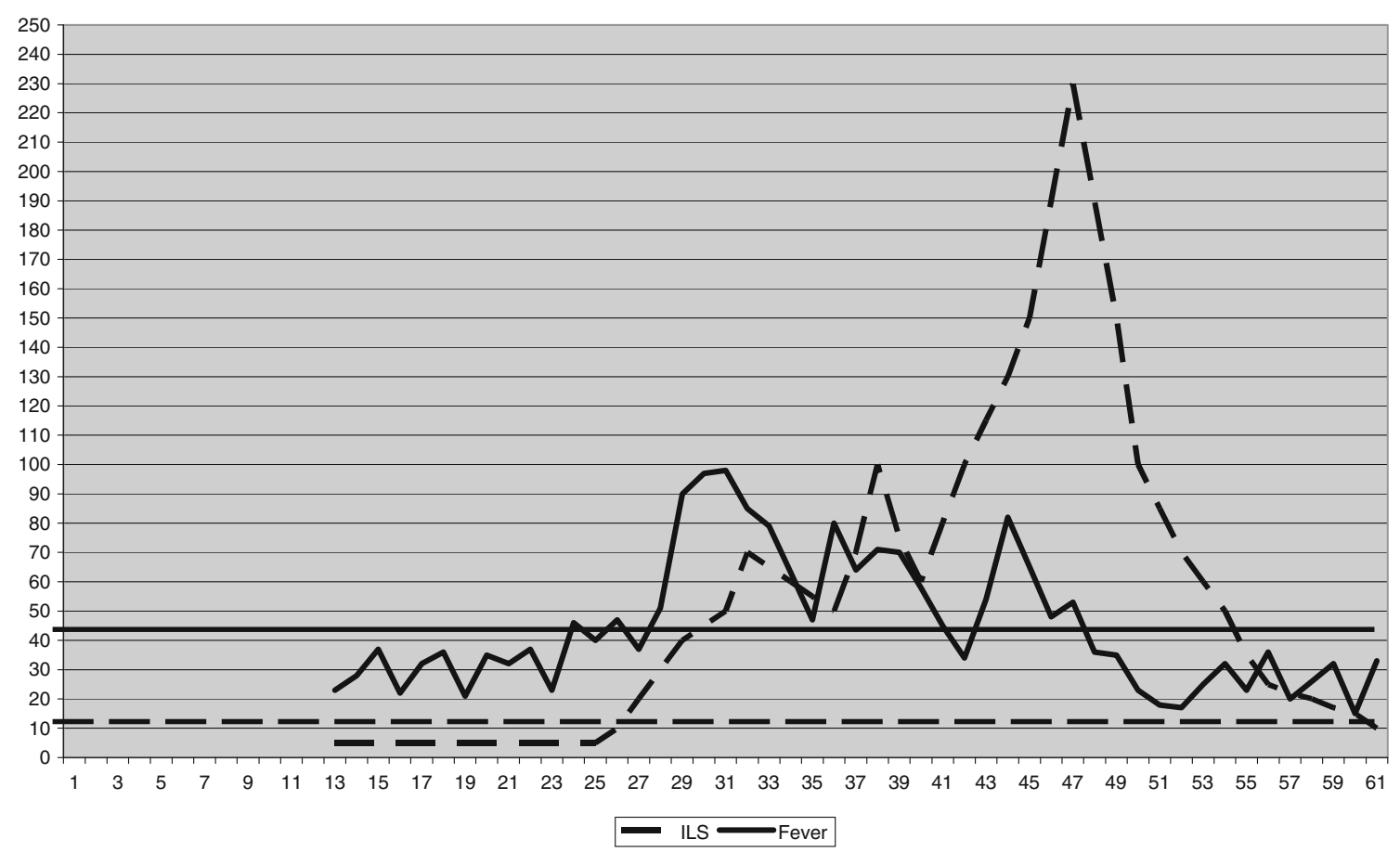

Fig. 1 Increased frequency of fever complaints during week 24 in the ED (rate is number per week), 2 weeks before an increase of influenzalike symptoms seen in the sentinel clinics (rate is number per 100,000). Areas under thick straight lines represent background activity

analyses of telehealth data found that fever calls for 5-14year-old children provided a timely and unique description of the evolution of a national influenza outbreak in England [12]. In studies comparing those with and without positive cultures for influenza virus, fever has been shown repeatedly to have better differentiating ability in comparison with other symptoms or combination of symptoms [18-21]. Also, patient self-reporting in the ED has been shown to be better than the chief complaint found in the medical chart for predicting the diagnosis made in the emergency room [22].

Extrapolation of our results to other settings should be done with caution. The frequency of presenting reasons in various ED settings is likely to vary significantly depending on socioeconomic factors such as type of hospital, lack of health insurance, younger age, unemployment, homelessness, and the lack of a primary care physician [23, 24]. Israel has comprehensive medical coverage for all, including family medicine services, and additional fees are levied on patients who attend the ED without a physician's referral and who are not subsequently hospitalized. Our hospitalization rate was $53.4 \%$, whereas rates of $34-71 \%$ $[23,24]$ have been reported in the USA and France, with the proportion of nonurgent visits ranging from 5\% to $82 \%$ in the USA [23] and 28\% to $41 \%$ in Europe [24].

We conclude that, in Israel, excess numbers of patients with a chief complaint of fever recorded by the admission secretary can predict increased influenza activity. Large comparative studies in various ED settings are warranted to determine if our findings can be extrapolated to other settings. If results of studies on Internet search engines are confirmed $[15,16]$, then they can be used in conjunction with selective viral surveillance to provide optimal, costeffective public health. The use of the ED secretary to record the chief complaint into an existing electronic database is an acceptable alternative if needed.

\section{References}

1. Hanratty B, Robinson M. Coping with winter bed crises. New surveillance systems might help. BMJ. 1999;319:1511-2.

2. Olson DR, Heffernan RT, Paladini M, Konty K, Weiss D, Mostashari F. Monitoring the impact of influenza by age: emergency department fever and respiratory complaint surveillance in New York City. PLoS Med. 2007;4:e247.

3. Dab W, Quenel P, Cohen JM, Hannoun C. A new influenza surveillance system in France: the Ile-de-France, "GROG". 2. Validity of indicators (1984-1989). Eur J Epidemiol. 1991; 7:579-87.

4. Quernel P, Dab W, Hannoun C, Cohen JM. Sensitivity, specificity and predictive values of health service based indicators for the surveillance of influenza A epidemics. Int J Epidemiol. 1994; 23:849-55

5. Silka PA, Geiderman JM, Goldberg JB, Kim LP. Demand on ED resources during periods of widespread influenza activity. Am J Emerg Med. 2003;21:534-9.

6. Irvin CB, Nouhan PP, Rice K. Syndromic analysis of computerized emergency department patients' chief complaints: an 
opportunity for bioterrorism and influenza surveillance. Ann Emerg Med. 2003;41:447-52.

7. Brillman JC, Burr T, Forslung D, Joyce E, Picard R, Umland E. Modeling emergency department visit patterns for infectious disease complaints: results and application to disease surveillance. BMC Med Inform Decis Mak. 2005;5:4.

8. Shimoni Z, Niven M, Kama N, Dusseldorp N, Froom P. Increased complaints of fever in the emergency room can identify influenza epidemics. Eur J Intern Med. 2008;19:494-8.

9. Daily L, Watkins RE, Plant AJ. Timeliness of data sources used for influenza surveillance. J Am Med Inform Assoc. 2007;14: 626-31.

10. Sloane PD, MacFarquhar JK, Sickberi-Bennet E, Akers R, Weber DJ, Howard K. Syndromic surveillance for emerging infections in office practice using billing data. Ann Fam Med. 2006;4:351-8.

11. Besculides M, Heffernan R, Mostashari F, Weisse D. Evaluation of school absenteeism data for early outbreak detection, New York City. BMC Public Health. 2005;5:105.

12. Cooper DL, Smith GE, Regan M, Large S, Groenewegen PP. Tracking the spatial diffusion of influenza and normovirus using telehelath data: a patiotemporal analysis of syndormic data. BMC Medicine. 2008;6:16.

13. Paget J, Marquet R, Meijer A, van der Velden K. Influenza activity in Europe during eight seasons (1999-2007): an evaluation of the indicators used to measure activity and an assessment of the timing, length and course of peak activity (spread) across Europe. BMC Infect Dis. 2007;7:141.

14. Brownstein JS, Freifeld CC, Madoff LC. Digital disease detection-harnessing the web for public health surveillance. N Engl J Med. 2009;360:2153-7.

15. Ginsberg J, Mohebbi MH, Patel RS, Brammer L, Smoliinski MS, Brilliant L. Detecting influenza epidemics using search engine query data. Nature. 2009;457:1012-4.
16. Polgreen PM, Chen Y, Pennock DM, Nelson FD. Using internet searches for influenza surveillance. Clin Infect Dis. 2008;47: 1443-8.

17. Bromberg M, Kaufman Z, Mandelboim M, Sefty H, Shalev V, Marom R. Clinical and virological surveillance of influenza in Israel-implementation during pandemic influenza. Harefuah. 2009;148:577-82.

18. Navarro-Mari JM, Perez-Ruiz M, Cantudo-Munoz P, Petit-Gancedo C, Jimenez-Valera M, Rosa-Fraile M. Influenza surveillance network in Andalusia. influenza-like illness criteria were pooly related to laboratory-confirmed influenza in a sentinel surveillance study. J Clin Epidemiol. 2005;58:275-9.

19. Zambon M, Hays J, Webster A, Newman R, Keene O. Diagnosis of influenza in the community: relationship of clinical diagnosis to confirmed virological, serologic, or molecular detection of influenza. Arch Intern Med. 2001;161:2116-22.

20. Carrat F, Tachet A, Rouzioux C. Evaluation of clinical case definitions of influenza: detailed investigation of patients during the 1995-1996 epidemic in France. Clin Infect D. 1999;28: 283-90.

21. Govaert TM, Dinant GJ, Aretz K, Knottnerus JA. The predictive value of influenza symptomatology in elderly people. Fam Pract. 1998;15:16-22.

22. Bourgeois FT, Porter SC, Valim C, Jackson T, Cook EF, Mandl KD. The value of patient self-report for disease surveillance. J Am Med Inform Assoc. 2007;14:765-71.

23. Gill JM. Non-urgent use of the emergency department: appropriate or not? Ann Emerg Med. 1994;24:953-7.

24. Lang T, Davido A, Diakite B, Agay E, Viel JF, Flicoteaux B. Non-urgent care in the hospital medical emergency department in France: how much and which health needs does it reflect? J Epidemiol Commun Health. 1996;50:456-62. 